symptoms could only be found in patients with an established diagnosis of SLE. In addition, a few rheumatologists were concerned about labeling Type 2 symptoms as 'SLE' since they attributed these symptoms to co-morbid conditions. Conversely, two providers noted that Type 2 symptoms could sometimes be part of Type 1 SLE activity.

Conclusion The Type $1 \& 2$ SLE model was well accepted by both patients and rheumatologists and considered as a useful approach to identifying and treating SLE manifestations. The concern that SLE could be mis-diagnosed in patients with only Type 2 symptoms indicates the importance of limiting the use of this model to patients meeting classification criteria for SLE. Many patients and several physicians suggested that the connection between Type 1 and 2 SLE may reflect an inflammation-driven subset of Type 2 symptoms.

\section{LONG-TERM OCULAR SAFETY OF HYDROXYCHLOROQUINE IN PATIENTS WITH CHILDHOOD-ONSET SLE (CSLE) FOLLOWED INTO ADULTHOOD}

${ }^{1}$ Wei Chen, ${ }^{2}$ Joanne Bargman, ${ }^{2}$ Murray Urowitz, ${ }^{3}$ Amanda Steiman, ${ }^{2}$ Dafna Gladman, ${ }^{2} J o r g e$ Sanchez-Guerrero, ${ }^{2}$ Zahi Touma, ${ }^{1}$ Deborah Levy, 'Linda Hiraki, 'Andrea Knight, ${ }^{1}$ Earl Silverman*. 'SickKids Hospital; ${ }^{2}$ University Health Network; ${ }^{3}$ Mount Sinai Hospital, University of Toronto, Canada

\subsection{6/lupus-2021-lupus21century.59}

Background In recent years there has been increasing concerns about the long-term ocular toxicity of hydroxychloroquine which has resulted in increased vigilance of the use of a maximum of $5 \mathrm{mg} / \mathrm{kg} /$ day. The aim of this study was to determine the long-term ocular safety of the use of hydroxychloroquine in patients with cSLE.

Methods The SLE Clinic database at SickKids hospital was searched for all patients followed since its inception in 1985 diagnosed and followed who were treated with hydroxychloroquine. Follow-up data was obtained into adulthood by reviewing the records of patients following transfer to care at an adult SLE clinic. While treated at SickKids all patients were treated with hydroxychloroquine at a dose $5.5-6 \mathrm{mg} / \mathrm{kg} /$ day (maximum $400 \mathrm{mg} /$ day).

However, following the recent recommendations for lower dosing of hydroxychloroquine, some of the patients had their dose lowered while followed in adulthood. All patients had regular hydroxychloroquine ophthalmologic follow-up at recommended intervals of 6-12 months. Exclusion criteria were: cSLE not diagnosed at SickKids Hospital, <2 follow visits, no documentation of ophthalmology follow-up or no documentation of hydroxychloroquine dose. This study was approved at local ethics boards at all participating centers.

Results A total of 718 patients with cSLE diagnosed until the end of 2019 were found in the SickKids Clinic database. 15 were not diagnosed with cSLE at SickKids or had $<2$ followup visits; 4 were eliminated as the hydroxychloroquine was stopped within one month for systemic side-effects. The study cohort there consisted of 699 patients who were followed for a total of 5815.5 person years. The mean follow-up time was 8.33 years (SD 6.15 years) (minimum 0.2 years and maximum of 35.9 years). $456 / 699$ (65\%) had $\geq$ 5year follow-up, 218 $(31 \%) \geq 10$ years; and $41(6 \%) \geq 20$ years. During the follow-up time one patient stopped hydroxychloroquine for deposition in the retina without visual changes. A second patient had hydroxychloroquine stopped based on an optometrist's examination but when reviewed by an ophthalmologist, no retinal changes were noted.

Conclusions In this long-term follow-up study of patients with cSLE treated hydroxychloroquine at a dose of $5.5-6.0 \mathrm{mg} / \mathrm{kg} /$ day (maximum $400 \mathrm{mg} /$ day) there was no evidence of visual changes after long-term follow-up of 5815.5 person years (patients followed for up to 35.9 years). We suggest that, unlike the potential for ocular toxicity of hydroxychloroquine found it studies of adult at doses $>5 \mathrm{mg} / \mathrm{kg} / \mathrm{day}$, patients with cSLE can be treated with $6 \mathrm{mg} / \mathrm{kg} / \mathrm{day}$ prior to transfer to adult care.

\section{SLE PHENOTYPES FORMED FROM MACHINE LEARNING AND THEIR ASSOCIATIONS WITH COGNITIVE IMPAIRMENT}

${ }^{1}$ Michelle Barraclough, 'Lauren Erdman, ${ }^{1}$ Andrea Knight, ${ }^{1} J u a n$ Pablo Diaz-Martinez, ${ }^{1}$ Kathleen Bingham, ${ }^{2}$ Jiandong Su, ${ }^{2}$ Mahta Kakvan, ${ }^{1}$ Maria Carmela Tartaglia, 'Lesley Ruttan, ${ }^{1} J o a n$ Wither, ${ }^{3}$ May Y Choi, ${ }^{3}$ Marvin J Fritzler, ${ }^{2}$ Dennisse Bonilla, ${ }^{1}$ Dorcas Beaton, ${ }^{4}$ Ben Parker, ${ }^{1}$ Robin Green, ${ }^{5}$ Patti Katz, ${ }^{4}$ lan N Bruce, 'Zahi Touma*. ${ }^{1}$ University of Toronto; ${ }^{2}$ University Health Network; ${ }^{3}$ University of Calgary; ${ }^{4}$ University of Manchester, ${ }^{5}$ University of California San Francisco

\subsection{6/lupus-2021-lupus21century.60}

Background Cognitive impairment (CI) in SLE is highly prevalent. Several factors are associated with CI: depression, pain, fatigue, medications, as well as more specific SLE factors such as disease damage, and autoantibodies. We aimed to phenotype CI in SLE using machine learning techniques to enable personalised targeted treatments.

Methods SLE patients aged 18-65 years attending a single completed the ACR Neuropsychological Battery (ACR-NB) cognitive assessment. Z-scores on all 19 tests of ACR-NB. ACR-NB tests were reduced using principal component analysis (PCA) to generate a factor score (CI Factor Score).

Demographic, clinical data, and patient reported outcomes including, SF-36, LupusQoL, the PDQ-20 (perceived cognitive deficits), Beck Depression Inventory-II, Beck Anxiety Inventory, and the fatigue severity scale (FSS) were analysed using similarity network fusion (SNF) to identify patient subtypes. Differences between the SNF identified subtypes were evaluated using Kruskal-Wallis tests and chi-square tests.

Results Of 301 patients, 89\% were women, mean age and disease duration at study visit $40.9 \pm 12.1$ years. The CI Factor score accounted for $28.8 \%$ of the variance and was associated predominantly with executive function and verbal memory. The SNF defined three subtypes (1, 2 and 3 with 60, 112, and 129 patients respectively) with distinct patterns in healthrelated quality of life (HRQoL), depression, anxiety, fatigue, fibromyalgia, medication usage, and damage. The CI Factor Score was significantly different between the subtypes. Examining specific cognitive domains revealed the most significant differences in the language processing and executive function tests. Subtype 3 performed worst on the majority of cognitive domains). Further exploration revealed statistical differences with depression, anxiety, fatigue, and fibromyalgia between the subtypes (figure 1). Differences were also found relating to organ involvement within the last ten years and damage within specific organs. No differences were found for SLE disease activity. Subtype 3 had higher levels of all conditions and 